described, with some heterogeneity being observed across monocyte subsets. However, although previously linked to blood pressure control, the significance of ACE expression on monocytes remains obscure.

Objectives: to evaluate whether monocyte subsets and ACE expression were associated with surrogates of CV subclinical disease in very early RA patients.

Methods: patients were recruited upon early referral to the rheumatology department. All patients were untreated at the time of sampling. The frequency of classical (CD14+CD16-), intermediate (CD14+CD16+) and nonclassical (CD14lowCD16+) monocyte subpopulations and ACE expression were assessed by flow cytometry in peripheral blood. Plaque occurrence, cIMT and stiffness parameters were analyzed by Doppler ultrasound in internal carotid, middle cerebral and basilar arteries.

Results: 53 patients were recruited, 47 fulfilling 2010 ACR/EULAR classification criteria for RA (36 women, $26 \mathrm{FR}+$ and $26 \mathrm{ACPA}+$ ) and 6 fulfilling criteria for Clinically Suspect Arthralgia (CSA) (6 women, $3 \mathrm{RF}+$ and 2 ACPA+). Non-classical and intermediate monocytes were positively associated with $\operatorname{cIMT}(r=0.413, p=0.005$ and $r=0.353, p=0.018$, respectively), as well as with pulsatility $(r=0.332, p=0.026$ and $r=0.323, p=0.030)$ and resistivity indices $(r=0.286, p=0.050$ and $r=0.277, p=0.045)$ at the left internal carotid artery. Expansion of CD16+ monocytes was not associated with disease activity, measured as DAS28 or SDAl (all p>0.050), but frequency of intermediate monocytes paralleled duration of the symptoms $(r=0.324, p=0.020)$.

ACE expression was higher in CD16+ monocyte subsets than in their classical counterparts. ACE expression was not associated with blood pressure, individual traditional CV risk factors, body mass index nor with mSCORE (all $\mathrm{p}>0.050$ ). ACE expression on intermediate and classical monocytes was correlated with cIMT $(r=0.382, p=0.010$ and $r=0.349$, $\mathrm{p}=0.019$, respectively). Moreover, ACE expression in all monocyte subsets was strongly associated with resistivity and pulsatility indices at the internal carotid artery (all $p<0.010$ )

Conclusion: the expansion of CD16+ monocyte subsets and the ACE expression are associated with arterial wall thickening and vascular functionality in treatment-naïve RA patients, hence suggesting a very early role for monocyte traits in this scenario.

None declared

DOI: 10.1136/annrheumdis-2019-eular.2641

\section{SAT0065 WNT5A INCREASES RHEUMATOID SYNOVIOCYTE MIGRATION THROUGH RYK RECEPTOR AND RHO- ROCK PATHWAY}

Angela Rodríguez-Trillo, Nerea Mosquera, Antonio Mera Varela, Antonio González, Carmen Conde. IDIS - CHUS Santiago de Compostela, Experimental and Observational Rheumatology Unit, Santiago de Compostela, Spain

Background: Fibroblast-like synoviocytes (FLS) are pivotal in inflammation and joint damage of rheumatoid arthritis (RA). These cells acquire an aggressive and invasive phenotype and secrete inflammatory mediators, metalloproteases and cathepsins that perpetuate inflammation and lead to cartilage and bone damage. Given the importance of FLS in the pathogenesis of RA, there is a growing interest in developing new therapies aimed to revert their aggressive phenotype. To this end, a better knowledgement of molecular mechanisms involved in FLS behavior is needed. Recently, the role of non-canonical Wnt pathway has been highlighted in several processes as migration, invasion and inflammatory responses. The non-canonical Wnt signaling pathway included the planar cell polarity (PCP), with the activation of Rho and Rac GTPases, and the Wnt/Ca2+ pathways. We have previously demonstrated that Wnt5a contributes to the aggressive phenotype of FLS by increasing their migration and invasion ability, and by stimulating the inflammatory response.

Objectives: To analyse the molecular mechanisms involved in the enhancing effects of non-canonical Wnt5a pathway on the aggressive phenotype of rheumatoid synoviocytes.

Methods: The expression of receptors of non-canonical Wnt5a pathway was assessed in FLS from six RA patients by real-time PCR. Expression of these receptors was suppressed by siRNA transfection (Dharmacon) and the antibody (anti-RYK, Abgent). Pharmacological inhibitors were used to identify the signaling pathways. Thus, an inhibitor of ROCK pathway (Y-27632, Selleckchem) was used and a calcium chelator (BAPTAAM, Enzo) for the inhibition of the PKC pathway. The expression of MAPKs was analyzed by western blot. Migration after stimulation with recombinant Wnt5a protein was analyzed by the wound healing assay using Ibidi inserts. The occupation area was determined by Image $\mathrm{J}$ software.

Results: We analyzed the expression of Wnt5a receptors, FZD1, FZD2, FZD4, FZD5, FZD7, ROR1, ROR2 and RYK of RA FLS. Although all receptors were expressed in theses cells, whereas ROR2 was weakly expressed, RYK showed the highest expression. Migration assays after receptor suppression by siRNA transfection or blocking antibodies showed the involvement of RYK on Wnt5a signaling in RA FLS. In effect, Wnt5ainduced migration was reduced by more than $25 \%$ in FLS treated with the anti-RYK antibody. In addition, Wnt5a-induced migration in FLS treated with ROCK inhibitor (Y-27632) was 32\% lower than in FLS not treated. No change in spontaneous migration was observed in FLS treated with Y-27632. However, the treatment with the calcium chelator, BAPTA-AM, did not show any differential effect between the spontaneous or the Wnt5a-induced migration. In addition, we found the activation of MAP kinases, ERK and P38, as well as, AKT and GSK3 $\beta$ in FLS treated with rWnt5a. Nevertheless rWnt5a did not activate JNK. These effects were reversed after treatment with the ROCK inhibitor or after blocking the RYK receptor.

Conclusion: These results indicate that Wnt5a contributes to the aggressive phenotype of RA FLS by binding to RYK receptor, through RhoROCK pathway and the activation of MAPKs, ERK and P38, as well as the activation of AKT and GSK3 3 .

Acknowledgement: Supported by grant of the ISCIII/PI17/01660/RETICS Program, RD16/0012/0014/Cofinanced FEDER

Disclosure of Interests: None declared

DOI: 10.1136/annrheumdis-2019-eular.4720

\section{SAT0066 DEFICIENCY OF TRANSCRIPTIONAL FACTOR T-BET ACCELERATES THE DEVELOPMENT OF COLLAGEN- INDUCED ARTHRITIS BY INDUCING T HELPER TYPE 17 DIFFERENTIATION}

Masaru Shimizu, Yuya Kondo, Kotona Furuyama, Masahiro Yokosawa,

Hiroto Tsuboi, Isao Matsumoto, Takayuki Sumida. University of Tsukuba, Tsukuba, Japan

Background: The transcriptional factor T-bet is required for Th-1 differentiation and important for regulating Th-17 differentiation, which plays a critical role in the development of collagen-induced arthritis (CIA). Previous studies reported that overexpression of T-bet suppresses Th-17 differentiation via inhibition of retinoic acid-related orphan receptor (ROR) $\gamma t$ and aryl hydrocarbon receptor $(A h R)(1,2)$. However, the function of $T$ bet in the development of CIA remains largely unknown.

Objectives: To clarify the role of T-bet on the pathogenesis of $\mathrm{CIA}$

Methods: 1) T-bet knockout (T-bet ${ }^{-1}$ ) mice (C57BL/6, B6 background) were generated as previously described (3). $\mathrm{T}^{- \text {bet }^{-1 /}}$ mice were immunized with CII with CFA, and the incidence and severity of CIA was assessed. 2) Anti-Cll antibodies in the sera from $\mathrm{T}_{\text {-bet }}{ }^{-\gamma}$ mice on day 60 after the first immunization were measured. 3) Lymph node cells from B6 or Tbet $^{-/-}$mice immunized with Cll were analyzed by flow cytometry using antibodies specific for CD3, CD4, CD8, CD11b, CD11c, CD19, Gr1 and NK1.1. 4) The lymph node cells described above were cultured in the CII containing medium, then the cytokine production was assayed by ELISA.

Results: 1) CIA developed in the $\mathrm{T}^{- \text {bet }^{-1}}$ mice was more severe than that of B6 wild-type mice and the incidence of arthritis was around 1.5fold higher. 2) The levels of total IgG, IgG1, IgG2b, IgG3 antibodies against native chicken $\mathrm{Cll}$ were significantly higher in $\mathrm{T}^{-\mathrm{bet}^{-1}}{ }^{-1}$ mice. How ever, IgG2a antibody production was significantly lower in $\mathrm{T}^{- \text {bet }^{-/}}$mice compared to $\mathrm{B} 6$ mice. 3) The number of $\mathrm{CD} 4+\mathrm{T}(\mathrm{CD} 3+\mathrm{CD} 4+), \mathrm{CD} 8+\mathrm{T}$ (CD3+CD8+), B (CD19+), dendritic cells (CD11C+), macrophage (CD11CCD11b+Gr1-), neutrophil (CD11c-CD11b+Gr1+), NK cell (CD3-NK1.1+) in the lymph node was not different but the number of NKT (CD3+NK1.1+) cells was significantly decreased in $\mathrm{T}_{\text {-bet }} /{ }^{-1}$ mice.

4) The level of IL-17 and IL-22 in the culture supernatant was significantly higher in $\mathrm{T}^{- \text {bet }^{-1}}$ mice, whereas that of IFN $\gamma$ was comparable in the two groups.

Conclusion: $\mathrm{ClA}$ was significantly severe in $\mathrm{T}^{- \text {bet }^{--}}$mice. The mechanisms of severe arthritis might be mediated through the up-regulation of IL-17 and IL-22 by Th-17 differentiation in the T-bet deficient and IFN $\gamma$ independent manner or the decrease of NKT cells.

\section{REFERENCES}

[1] Kondo $\mathrm{Y}$, et al. Overexpression of T-bet gene regulates murine autoim mune arthritis. Arthritis Rheum 2012;64:162-72 